\title{
Pilot research of women's codependent behavior using Rorschach test
}

\author{
Zvulun Bar-On ${ }^{1}$, Alisa Achina ${ }^{2}$, Anastasia Kolenova ${ }^{2 *}$, Ekaterina Denisova ${ }^{2}$ \\ ${ }^{1}$ Sadot Institute, Tel Aviv, Israel \\ ${ }^{2}$ Don State Technical University, Faculty "Psychology, Pedagogy and Defectology", \\ 344000 Rostov-on-Don, Russian Federation
}

\begin{abstract}
One of the most famous projective techniques around the world - G. Rorschach inkblot test. In many countries this technique is rather popular because it can give the chance of both the qualitative and quantitative analysis. This thesis is confirmed in research works of J. Eksner Institute. For several decades J. Eksner Institute (USA) have been collected psychometric data with using Rorschach test in various groups of the population. These studies make it possible to conduct a clinical and psychological studies and determine the mental status of the subject. Our research is devoted to informational content of Rorschach test of women who have difficulties in interpersonal interaction within their family (with an addictive partner). It was shown that with increase of manifestation intensity of codependent behavior also the orientation of codependent women in interpersonal interaction changes, the coding symbols (determinants), specific for the codependent women, in answers to the third table of Rorschach test (an integrative system of J. Eksner) were also revealed. The practical relevance of this research is that the obtained results can form a basis of express diagnostics of the women having difficulties in interpersonal interaction within the family (husbands, children).
\end{abstract}

Codependent behavior is understood as the learned set of behavioral patterns of self-defeating behavior which leads to reduced ability to initiate and participate in social relationship [1-4], and today in the work of the clinical psychologists it takes a very important place. For the last several decades the problem of codependent behavior us has been one of the leading subject in psychological work with clients [5-8]. In this regard the pilot research conducted is rather relevant. In this study we try to define specific features and the general regularities of answers of codependent women on the third table of G. Rorschach inkblot test (interpretation of data was carried out on the basis of the content analysis as well as using the complex J. Ekser system).

Methodical tools:

1. Test "A scale of measurement of codependence in the relations of Spanna-D. Fisher" (in V.D. Moskalenko's adaptation) [9, 10];

\footnotetext{
* Corresponding author: kolenova.nastya@,yandex.ru
} 
2. Method "Write the story" (of V. Lanovoy using stimulating material - the third table of Rorschach inkblot test $[11,12]$.

Test "A scale of measurement of codependence in the relations of Spanna-D. Fisher" (in V.D. Moskalenko's adaptation) - is the tool helping individuals and professionals of the assisting professions to receive fast diagnostics of codependent behavior. This test was developed to help people to define not only whether they are codependent, but also in what degree.

G. Rorschach inkblot test is a psychological test where subjects' perception of the inkblots is registered and then analyzed with psychological interpretation. Clinical psychologists use this test for studying personal traits and emotional functioning of a person. It was used for identification of the main disorder of thinking, especially when patients do not want to describe the thought processes openly.

In this study, we used only the third table of the test of Rorschach as stimulus material as it has semantic idea "interpersonal relations".

Test "A scale of measurement of codependence in the relations of Spanna-D. Fisher" (in V.D. Moskalenko's adaptation) was used for solving the first task. [9, 10]. Therefore there was an opportunity to divide all respondents into subgroups: norm -11 people (a $\leq 32 \mathrm{sc}$.), moderate codependence -85 people $(33 \mathrm{sc} . \leq \mathrm{a} \leq 60 \mathrm{sc}$.) and pronounced codependence -22 persons (61 sc. $\leq \mathrm{a} \leq 96$ sc.). Subgroup "moderate codependence" was divided into 2 subgroups: "the moderate codependence gravitating to the lower bound" - 48 people (33 sc. $\leq \mathrm{a} \leq 45 \mathrm{sc}$.) and "the moderate codependence gravitating to the upper bound" -37 people (46 sc. $\leq \mathrm{a} \leq 60$ sc.).

To solve the second task, it was offered to respondents to write the story consisting of 57 sentences, having looked at the third table of Rorschach test. Analytical and statistical data processing of the content analysis was carried out using the program of data processing Statistica 12.

For the solution of the third task the received stories were coded on a system of J. Eksner [13], and the results were subject to the frequency analysis of data.

Results:

1. The received protocols while carrying out the method of "Write the story" of presentation of the third table of Rorschach test were coded by rules of the Integrative system of J. Eksner [13, 14], and also underwent the frequency analysis. In this regard the frequency of sign occurrence was most brightly shown in the following of 14 of 51 coding characters (determinants) $(\mathrm{p} \leq 0,05)$ (Fig. 1):

$\mathrm{Ma}$ is the active human movement. It is used for the answers including physical human activity (to run, speak, etc.) or similar to that activity of animal and fictional characters;

FMa is the active movement of animals. It is used for the answers including physical activity of animals. The apprehended movement has to coincide with a kind of an animal. When action of an animal does not coincide with his nature, the answer is coded as M;

$\mathrm{FC}$ is a form color. It is used for answers which are created generally on features of a form. Color is used, but it is less important;

(2) - couples. It is used for answers in which it is reported about two identical objects, according to symmetry of a spot. Objects have to be equal in every respect, but should not be defined as reflections or an object and its image in a mirror;

Fr is a form reflection. It is used for answers in which the spot or its part are defined as reflection or the image in a mirror, based on symmetry of a spot. An answer essence is in features of a form, and the called objects meet the requirements of a certain form;

$\mathrm{H}$ - for the answers including the person entirely;

$(\mathrm{H})$ - for the answer, including the person entirely in a fictional or mythical image (the fairy, a giant, an angel, etc.); 
$\mathrm{Hx}$ - is usually coded as the second contents in answers where human emotions or feelings and experiences are obviously attributed to objects. Attributing of emotion or experience has to be clear and unambiguous;

Art - for the answers containing cultural implication: painting, drawing, works of art, illustrations, etc;

$\mathrm{Fd}$ - for any edible or suitable in food to people of objects;

$\mathrm{P}$ - popular answers, coded according to the table of the popular answers given on an ink spot;

$\mathrm{COP}-$ are joint actions, movements;

GHR - is good attitude to people;

PHR - is the bad attitude towards people.

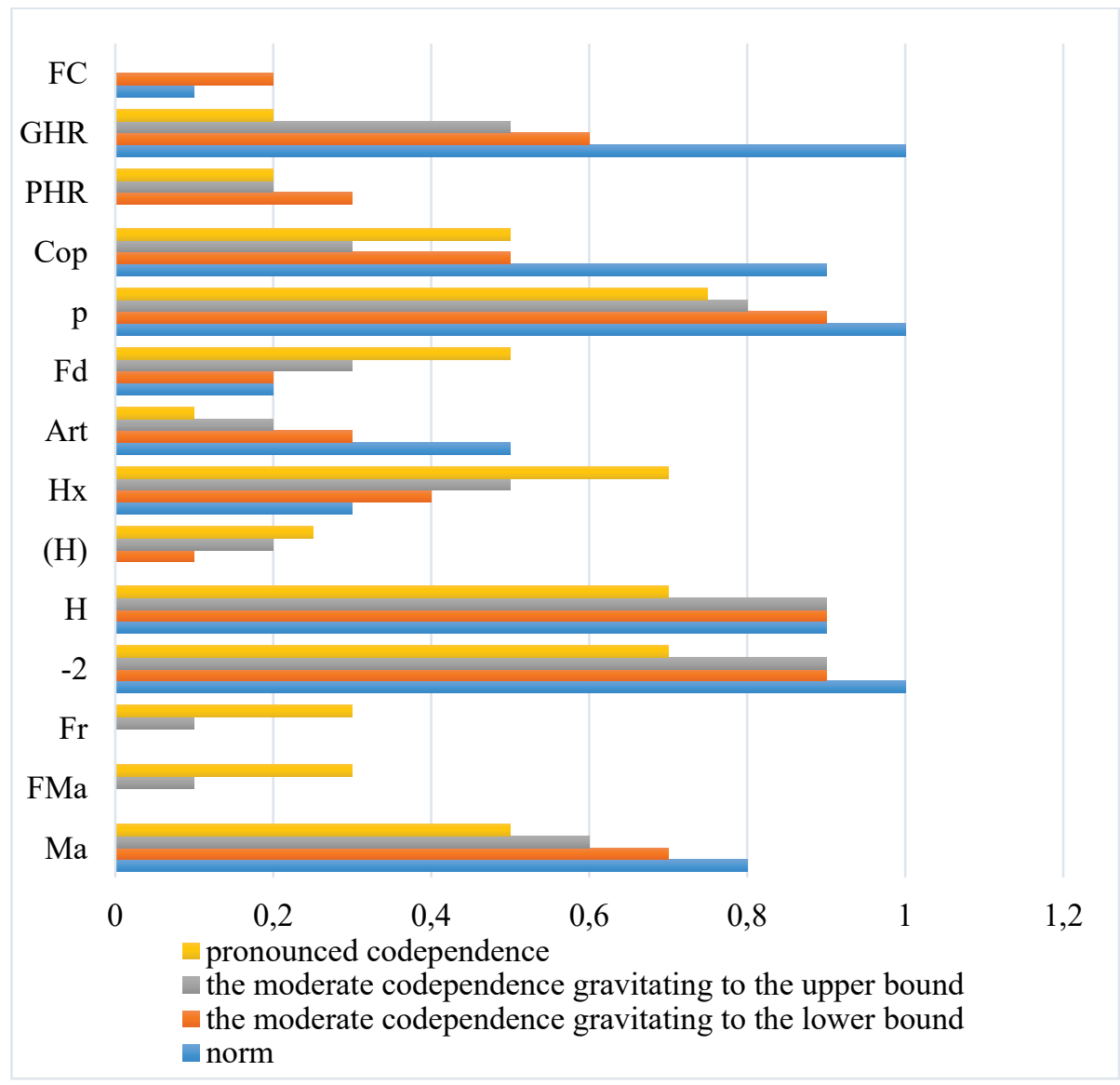

Fig 1. The indicators in a cluster "Content" revealed by means of the III table of G. Rorschach test in interpretation on the Integrative system of J. Eksner. Statistically significant differences between groups with codependent behavior by Kruskal-Wallis's criterion.

So, increase by sign the active human movement (Ma) from subgroup of codependence to control group confirm decrease in adaptability of respondents in connection with increase of signs of codependent behavior $(\mathrm{p} \leq 0,1)$.

Existence of couples (2) show adequate interaction with them testify to a possibility of creation of communications with other people, this indicator decreases towards growth of codependent behavior that means decrease of this skill $(\mathrm{p} \leq 0,05)$. 
At the same time the indicator of fictional people $((\mathrm{H}))$ grows that speaks about increase of a habit of attributing to other people, nonexistent lines in process of increase on a codependence scale $(\mathrm{p} \leq 0,05)$.

Reduction of the index of intellectual protection (Art) demonstrates weakening of the highest protective mechanisms in process of increase of codependence that in itself conducts to loss of adequacy of response to the changing conditions, and taking into account previous two - to violation in adaptation $(\mathrm{p} \leq 0,01)$.

Decrease on a scale of interpersonal cooperation (Cop), demonstrates difficulties in cooperation, in interaction with people (An example illustration of a statement of the client: "I do not ask for help, nobody will do it better, than I will. But I accuse everybody of not helping me") $(\mathrm{p} \leq 0,01)$.

2 . The content analysis of the received protocols by a technique "Write the story" allowed to reveal the following orientations in subgroups of women with different manifestation of codependent behavior $[11,12]$ :

- orientation of activity on creation

- orientation of activity on an object

- orientation of activity at each other

- lack of the object relations

- realness

- not realness

- merge (loss of borders)

- need for approval

- perspective in time

- overcoming difficulties

The frequency analysis of expressiveness of scales in studying of orientations in interpersonal interaction received by means of the program of mathematical statistics Statistica $12(\mathrm{p} \leq 0,05)$ is provided on figure 2 :

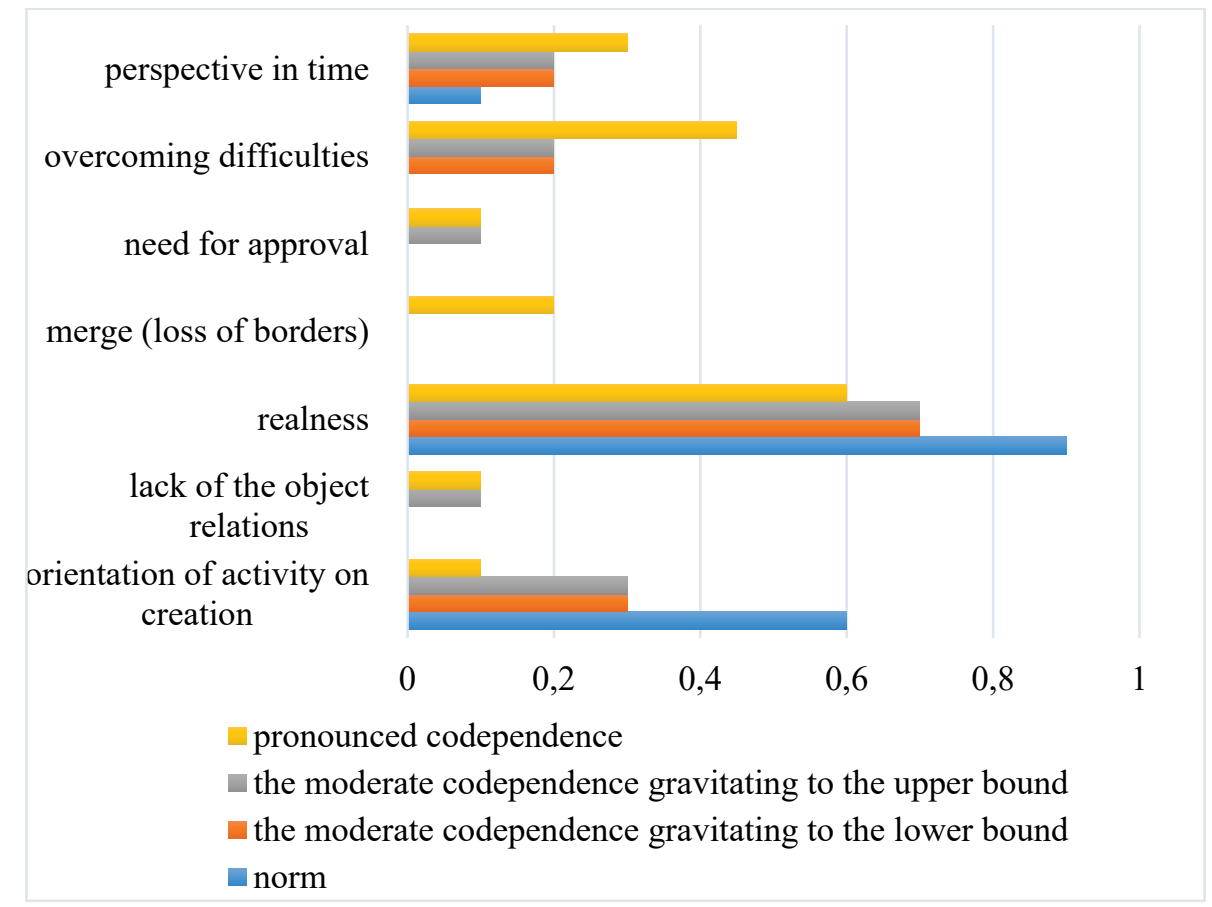

Fig 2. Orientation indicators of the codependent women in interpersonal interaction 
Degree of expressiveness revealed by the content analysis of orientation indicators of the codependent women in interpersonal interaction:

- orientation of activity on creation $(28.8 \%)$

- lack of the object relations $(5.1 \%)$

- realness $(70.3 \%)$

- merge (loss of borders) (4.2\%)

- need for approval (5.1\%)

- overcoming difficulties $(22.8 \%)$

- perspective in time (18.6\%)

We draw our attention to the fact that there are high-quality changes of the marked-out signs on several groups of regularities:

1 - in subgroup "Norm" the following orientations were revealed:

- "orientation of activity on creation" (high values);

• "realness" (high values);

- "orientation in time" (small values).

These signs give us the right to say that people with signs of codependent behavior at the Norm level in the interpersonal interaction lead to a possibility of establishment of the functional relations, with an opportunity to estimate events of the life is realistic and at the same time the orientation of patients is revealed to live, preferably, in the afternoon.

2 - in subgroup "Lower moderate level of codependence" except the above described orientations which began to be shown less. Orientation on "overcoming difficulties" was found in this subgroup of respondents.

Thus, we can assume that at the general opportunity to estimate realistic events around, ability to build the functional relations respondents from this group estimate the life as complicated by various tests and difficulties which demand their involvement.

3 - subgroup "upper moderate level of co-dependence "above described focus: "focus on creation", "realism" decreased, which indicates a decrease in the level of adequate perception of reality. Also, the above indicators "overcoming difficulties" and "orientation in time" remained unchanged, which indicates the similarity of the subgroups of the lower and upper moderate levels of co-dependence. At the same time, these respondents show new directions for a moderate level of co-dependent behavior: "lack of object relations" and "need for approval". Thus, we assume that these directions show that with an increase in the manifestation of co-dependent behavior, respondents in interpersonal interaction do not perceive the interlocutor empathically, do not take into account his wishes and needs, as well as interaction with others becomes driven by the desire to obtain approval from other people.

4 - in the subgroup "Highly pronounced co-dependence" there is a new direction "merge", which indicates that respondents from this subgroup tend to adjust to the other in interpersonal interaction, adopt his ideology to feel better contact with him. Taken into account that the trend: "focus on creation" and "realism" is reduced, it can be assumed that this pattern leads to a lack of functional relationships, which can serve as a reason for the treatment of a co-dependent patient to a therapist or psychologist, while the focus of "overcoming difficulties" increases sharply compared to other subgroups, this indicates the belief of respondents that life is complex and is perceived as a series of tests. The growth of the orientation "orientation in time" is also revealed, which indicates the perception of the respondents ' data of reality in anticipation of something better, so their life passes in dreams of a bright future, but they are not ready to change their present [15]. We can note that the following orientations: "the lack of the object relations", "merge", "requirement for approval" and "overcoming difficulties" are absent in subgroup norm and are shown at increase on a codependence scale that gives the chance to assume that these criteria can initially draw the attention of the specialist that the respondent has the tendency of codependent behavior. 
Thus, we conducted a comprehensive analysis of the use of the third table of $\mathrm{G}$. Rorschach's inkblot test for diagnostics of codependent women. The results obtained correspond to objectives and allowed us to describe personal characteristics of codependent women and also to the description of personal characteristics of codependent behavior. That gives us the opportunity to speak about legitimacy of the use of the third table of G. Rorschach inkblot test.

Thus, the use of the Rorschach test for clinical and psychological examination and identification of coding symbols gives a chance of performing express diagnostics among this category of women and this reveals the practical significance of our work.

This research was funded by the Russian Foundation for Basic Research (RFBR), project № 18-01301209.

\section{References}

1. V.D. Moskalenko, Dependence. Familial disease (2018)

2. M. Beattie, Beyond Codependency and getting better all the time (1989)

3. B. Carruth, W. Mendenhall, Co-dependency: Issues in Treatment and Recovery (1989)

4. C.Z. Whitefield, Alcohol. Treat. Quart.,19, 3- 17 (1989)

5. M. Leichtman, On preserving a legacy: Paul Lerner and the Menninger Rorschach tradition (2014)

6. R. Schafer, Tragic Knots in Psychoanalysis: New Papers on Psychoanalysis (2018)

7. N.A. Tsvetkova, A.I. Rybakova, Russian Psychological Journal, 15 (3), 116-142 (2018)

8. E. Young, J. Psychoactive Drugs, 19 (3), 257-268 (1987)

9. V.D. Moskalenko When there is too much love (2018)

10. J.L. Fischer, L. Spann, D. Crawford, Alcogolism. Treat. Quart, 8(1), 87-98 (1991)

11. E.V. Lanovoy, Method "Write a story: diagnosis, therapy and prognosis" (2006)

12. H. Rorschach Psychodiagnostics: A diagnostic test based on perception (1942)

13. J. Exner, Newsletter (E.R.A.), 14, 6-9 (2005)

14. M. Assanovich, The integrative system of psychodiagnostics by the Rorschach method (2011)

15. B. Smith. RORSCHAHIANA, 38 (1), 12-22 (2017) 